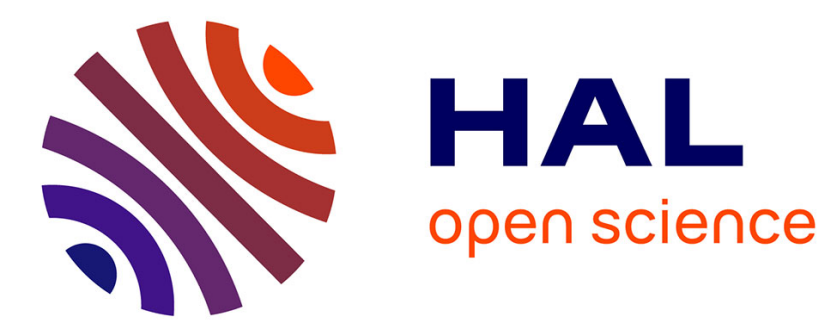

\title{
Les syndicats de dockers et la Fédération des Ports et Docks à l'épreuve du syndicalisme légal
}

\author{
Michel Pigenet
}

\section{To cite this version:}

Michel Pigenet. Les syndicats de dockers et la Fédération des Ports et Docks à l'épreuve du syndicalisme légal: (1940-1944). Michel Margairaz, Danièle Tartakowsky. Le syndicalisme dans la France occupée, Presses universitaires de Rennes, pp.213-224, 2008, Histoire. halshs-00779793

\section{HAL Id: halshs-00779793 \\ https://shs.hal.science/halshs-00779793}

Submitted on 22 Jan 2013

HAL is a multi-disciplinary open access archive for the deposit and dissemination of scientific research documents, whether they are published or not. The documents may come from teaching and research institutions in France or abroad, or from public or private research centers.
L'archive ouverte pluridisciplinaire $\mathbf{H A L}$, est destinée au dépôt et à la diffusion de documents scientifiques de niveau recherche, publiés ou non, émanant des établissements d'enseignement et de recherche français ou étrangers, des laboratoires publics ou privés. 


\title{
Les syndicats de dockers et la Fédération des Ports et Docks à l'épreuve du syndicalisme légal (1940-1944)
}

\author{
Michel Pigenet \\ Centre d'Histoire sociale du XXe siècle (Paris 1/CNRS)
}

\begin{abstract}
Á la veille de la guerre, la fédération des Ports et Docks se situe dans la mouvance de Syndicats. Sortis renforcés des grèves de 1936, les ex-unitaires ne sont pas parvenus à supplanter les «confédérés » et, moins encore, à entamer au Havre, la plus solide base fédérale, l'autorité des anciens autonomes de sensibilité syndicaliste-révolutionnaire ${ }^{1}$. En septembre 1938, 93 - $57 \%$ - de ses 161 syndicats représentés au congrès confédéral de Nantes soutiennent la motion Delmas sur la paix. Impulsée du sommet de la CGT, l'épuration anticommuniste est menée sans état d'âme sur les quais. Le 12 octobre, l'approbation de la position de la CA de Confédération par le comité national des Ports et Docks se double d'une vigoureuse dénonciation de "l'infâme trahison russe », «abominable attentat contre le prolétariat international». En l'absence d'un sursaut d'indignation des « dirigeants communistes de nos organisations », le comité vote leur exclusion. Répercutée à tous les échelons, la mesure ampute les directions syndicales de Bordeaux et Dunkerque, entraîne la désaffiliation d'autorité des structures récalcitrantes de Marseille et de Rouen ainsi que du syndicat général de la marine fluviale. Avant même les dissolutions judiciaires, la Fédération encourage la création de syndicats concurrents par les responsables écartés au lendemain de la poussée « unitaire » des années précédentes.
\end{abstract}

\section{La Fédération en question}

\section{Les ports dans la tourmente}

Si la drôle de guerre stimule un temps le trafic des ports, l'offensive allemande bouleverse la donne. A la mi-juin 1940, l'activité des sites de la façade occidentale est au plus bas. La rigueur et l'efficacité croissantes de la surveillance exercée sur mer par les Britanniques interdisent une franche reprise en-dehors du cabotage. Enjeux stratégiques, les ports de la zone occupée sont la cible des bombardements dont la fréquence s'intensifie au fil des mois au point de motiver la revendication, le 27 novembre 1941, d'une allocation spéciale pour les salariés de ces secteurs «particulièrement dangereux ». Á Dunkerque, déjà classée ville la plus sinistrée de France en 1940, la menace aérienne et les destructions amènent l'arrêt pur et simple des manutentions. Entre Dunkerque à Bordeaux, «il y a peut-être cent dockers qui travaillent encore », estime le secrétaire de la Fédération des Ports et Docks en novembre $1943^{2}$. Partout, le sous-équipement aggravé par les prélèvements de l'occupant et les mauvaises conditions de ravitaillement a pour effet une baisse sensible des rendements.

En zone occupée, le dirigisme vichyssois coexiste, tant bien que mal, avec les impératifs de l'effort de guerre allemand dont les divers commandements veillent à faire prévaloir la primauté. Sur le port de Bordeaux, la Haffenkommandantur, dépendante de

\footnotetext{
${ }^{1}$ Cf. la composition du bureau fédéral élu en 1938 où siègent Loriot (secrétaire, ex-confédéré), Le Gall (secrétaire, ex-autonome), Blanckaert (secrétaire, ex-autonome), Piquemal (trésorier, ex-confédéré), Filhol (secrétaire-adjoint, ex-confédéré), Philippe (secrétaire-adjoint, ex-unitaire) et Hauguel (trésorier-adjoint, exautonome).

${ }^{2}$ Archives nationales (AN), F22 1972, Comité social national - CSN- de la Famille des Transports, séance du 20 novembre 1943.
} 
Kriegsmarine, devient l'employeur principal et, surtout, prioritaire ${ }^{3}$. Mécontents, les Allemands envisagent de procéder à la réquisition des dockers qui leur sont nécessaires et de retirer aux entreprises leur traditionnelle fonction d'intermédiaires. Face à cette menace, le directeur et les employeurs obtiennent la création d'une sorte de pool : l'Office de répartition de la main-d'œuvre. Selon la logique de souveraineté chère aux autorités de Vichy, l'Office, présidé par un employeur auquel on adjoint le secrétaire du syndicat ouvrier, mutualise la fourniture des travailleurs exigés par l'occupant.

Après le débarquement allié en Afrique du Nord et l'occupation de la zone sud, les effectifs de dockers marseillais chutent à 700 en 1943, dont une centaine continue à se présenter à l'embauche. Dès leur arrivée, les Allemands s'empressent de superviser l'attribution des cartes d'accès au port, réquisitionnent engins et hangars. Une décision plus brutale et définitive se profile : la destruction du quartier Saint-Jean, près du Vieux Port, où résident de nombreux dockers. Á la suite d'attentats et d'une poussée des désertions, un télégramme en provenance de Berlin ordonne le «nettoyage » d'une zone d'habitat vétuste, réputée héberger une population pauvre et d'origine étrangère, suspecte d'activités illicites. Le 22 janvier, le déclenchement de l'opération d'évacuation de quelques 25000 personnes prépare les destructions à l'explosif opérées à partir du $1^{\mathrm{er}}$ février. Par-delà un radicalisme hygiéniste et sécuritaire obsessionnel, l'occupant entend disposer d'une zone littorale aménagée pour repousser un éventuel débarquement. Des mesures similaires, quoique moins spectaculaires, affectent d'autres sites et se multiplieront en 1944.

\section{La Fédération des Ports et Docks face aux replis corporatifs et locaux}

Investi en juin 1940, le siège de la Fédération, au 213 de la rue Lafayette, subi un pillage en règle. Hébergé à la Bourse du Travail, le secrétariat doit attendre mars 1941 pour reprendre possession de ses anciens bureaux. Délestée des 900000 francs en espèces «perdus » à la faveur de la débâcle, la trésorerie ne peut compter sur la perception de cotisations en chute libre. Á Paris, le secrétariat fédéral apprend à se débrouiller avec les maigres ristournes que lui versent les syndicats de la capitale. Contrainte aux économies, l'organisation revient sur la décision d'appointer Blanckaert, puis cesse de salarier Loriot ne conservant qu'un seul permanent: Le Gall ${ }^{4}$. Cette gêne fragilise l'architecture fédérale. Une première fracture professionnelle intéresse les syndicats de la batellerie dont les spécificités n'ont jamais vraiment retenu l'attention des dockers. Dès l'automne 1940, sur fond d'effondrement des disciplines de tendances, Philippe, Delval et Gau, unitaires en rupture d'affiliation, fondent une Fédération nationale de la navigation fluviale. Celle-ci aurait reçu l'adhésion du petit syndicat général de la batellerie artisanale qu'anime Louis Louis, un exconfédéré dont la frénésie épistolaire ne parvient pas à occulter la faiblesse rédhibitoire de sa vingtaine d'adhérents. Les recompositions consécutives à la définition des frontières des familles professionnelles privent encore la Fédération des Ports et Docks de l'ancienne section technique des usines et chantiers de charbon récupéré par son homologue du Sous-sol.

Dans un article aux allures d'appel à l'aide en vue de «reconstruire l'organisation professionnelle », Loriot traite des obstacles qui gênent, sinon empêchent, l'information des syndiqués ${ }^{5}$. Dépourvus de moyens de propagande en territoire occupé, ses camarades ne parviennent plus à organiser de réunions ou à placarder des affiches. En zone Sud, Blanckaert réussit à convoquer, fin 1941-début 1942, un congrès de la subdivision méditerranéenne, mais

\footnotetext{
${ }^{3}$ Archives de l'Institut CGT d'Histoire sociale (AICGTHS), Fonds Jayat, Comité nationale de reconstitution des organisations syndicales (CNROS), lettre du préfet de la Gironde au Président de la Commission, le 30 décembre 1944.

${ }^{4}$ Rapport financier de Piquemal. Congrès fédéral (19-22 mars 1946), Fédération des Ports et Docks, Salle Lancry, Paris, Imprimerie spéciale de la Fédération, p. 156.

${ }^{5}$ L'Atelier du 26 avril 1941.
} 
interprète à sa façon le mandat reçu de la Fédération. Lié au comité syndical de coordination de la zone sud, le batelier pense surtout à survivre. Dirigeant une coopérative, puis une entreprise du bâtiment, il ne répond plus aux demandes de règlement du matériel syndical expédié depuis Paris. L'édition par ses soins d'une carte fédérale pour la zone non occupée conforte certains militants dans le soupçon d'une dissidence. Malgré la protection de Loriot, Blanckaert devra démissionner du secrétariat en 1943 sous la pression de Le Gall et Hauguel. On sait moins de choses sur les circonstances exactes de l'éviction de Filhol dénoncé comme informateur du patronat ${ }^{6}$.

\section{Expédients et expériences originales}

Ici et là, des coopératives ouvrières de manutention servent de refuges aux militants sans troupes. Á Bordeaux, l'une d'elles, créée en 1939, ne s'active vraiment qu'à partir de 1941 sous la houlette de Loriot dont elle complète les revenus. Le Gall s'émeut toutefois de la mauvaise volonté des autorités portuaires qui, à Rouen, rechignent à leur louer l'outillage public $^{7}$. Partout, les syndicats ne négligent aucune occasion d'atténuer les rigueurs du temps. Celui de Rouen appuie, en décembre 1943, la création d'un comité social interentreprises. Le projet répond à la nature particulière de la manutention dont le gros de la main-d'œuvre se compose de salariés intermittents. Reprise et précisée par le directeur du port, la proposition inspire un rapport favorable à sa généralisation dans tous les sites.

Á Paris, Marcel Boucher reprend le travail de lobbying grâce auquel il avait obtenu, au printemps et à l'automne 1939, l'institution d'une carte professionnelle, puis la création, pour le port de Paris, d'un bureau de répartition de la main-d'œuvre ${ }^{8}$. Le dernier texte stipule le recrutement du personnel du nouvel organisme parmi les «organisations professionnelles dont l'existence aura été reconnue par le ministre ». Au même moment, Bordeaux inaugure un système voisin, sous l'impulsion de Louis Fischer, directeur du port autonome et prochain directeur des ports maritimes et des voies navigables. Satisfaits, les syndicalistes réclament, par la voix de Loriot, son extension à tous les ports. Ce que fera la loi du 28 juin 1941.

\section{Clivages anciens et nouveaux}

Après sa nomination, le 14 juillet 1940, au secrétariat d'État au Travail et à la Production industrielle, René Belin mise dorénavant sur la voie autoritaire du réformisme sans perdre le contact avec les anciens de Syndicats ${ }^{9}$. En avril 1941, Loriot lui en sait gré. Agacé par le retard de décisions attendues, il redit combien la présence au gouvernement de l'ancien secrétaire de la CGT «donne confiance » à ses camarades ${ }^{10}$. Á cette date, la dissolution des confédérations est effective depuis près de six mois. Aux dires ultérieurs de Le Gall, la Fédération des Ports et Docks aurait protesté contre la mesure et témoigné sa solidarité aux membres du bureau confédéral ${ }^{11}$. En sa qualité de secrétaire, lui-même signe, le 6 juin 1941, la déclaration des vingt-et-une fédérations ouvrières opposées à leur éventuelle dissolution et à la création d'organisations professionnelles mixtes. L'organisation apporte simultanément son concours au Comité syndical de coordination, maigre substitut béliniste de la CGT. En pointe sur cette ligne qu'il développe dans L'Atelier, Loriot convoque le passé, certifiant que les dockers «n'ont besoin de faire aucun effort pour s'adapter aux idées de la Révolution nationale », eux qui, « depuis toujours à l'avant-garde de la lutte pour le progrès social », ont

\footnotetext{
${ }^{6}$ Intervention de Gagnaire et de Hauguel au congrès fédéral de 1946, op. cit., p. 32-37, 156 et suivantes.

${ }^{7}$ AN, F22 1968, Commission provisoire d'organisation (CPO) des Transports, session du 24 février 1944.

${ }^{8}$ Décrets des 13 mai et 23 octobre 1939.

${ }^{9}$ Cf. J.-P. Le Crom, Syndicats, nous voilà. Vichy et le corporatisme, Editions de l'Atelier, 1995.

${ }^{10}$ L'Atelier du 26 avril 1941.

${ }^{11}$ Rapport au congrès fédéral de 1946, op. cit., p. 6 et intervention de Hauguel, p. 28.
} 
su déjouer les «traquenards» communiste ${ }^{12}$. Le rôle du syndicaliste bordelais décline, cependant, à partir du printemps 1942. L'effacement précède la perte du statut de permanent, en décembre 1943, et de plus sérieux déboires à la Libération où il sera brièvement arrêté. N'exagérons pas, pour autant, les différences perceptibles entre ses inclinations et celles de Le Gall. Ainsi est-ce sous le nom de celui-ci que la Fédération adhère, le $1^{\mathrm{er}}$ mai 1942, au texte commun à plusieurs organisations qui, «malgré quelques critiques justifiées », proclament leur «attachement» à la Charte. Fières des traditions du mouvement syndical, elles en rappellent les combats «pour le rapprochement des peuples », avant 1914, lors du traités de Versailles et de Munich. Pour l'heure, le manifeste condamne sans réserve « tous les attentats et les actes de violence qui ont pour but de jeter le trouble parmi le pays ».

\section{Syndicats et syndicalistes entre contraintes et horizons inédits La loi du 28 juin 1941 : les ambiguïtés d'une avancée sociale}

L'armistice ne met pas fin à la tutelle administrative instaurée, le 2 septembre 1939, sur les activités portuaires et maritimes. En plus de la réquisition de la flotte de commerce, les pouvoirs publics négocient avec les entreprises de manutention la fixation de tarifs plafonds. Ils se penchent aussi sur l'organisation de l'embauche et du travail, thème de la loi du 28 juin 1941. Selon Jean Berthelot, le texte fut préparé en concertation avec les employeurs et des syndicalistes ${ }^{13}$. Un premier projet de « service public de la manutention » se serait heurté à l'opposition discrète, mais ferme, du patronat dont le syndicat professionnel s'est aisément coulé dans les structures du comité d'organisation de la branche. Les employeurs, toujours un peu tâcherons, ne redoutent rien tant que d'être court-circuités. Un rapport inspiré de l'expérience bordelaise enregistre ces craintes, mais suscite celles de Loriot, inquiet de son examen par le comité d'organisation d'où les travailleurs sont exclus.

De fait, les treize articles de la loi établissent une priorité d'emploi aux dockers titulaires d'une carte professionnelle, classés et contrôlés dans chaque site par un bureau central d'embauche de la main-d'œuvre - BCMO - financé par les entreprises et le gestionnaire du port ${ }^{14}$. Sans surprise, l'avancée, objectif des plus anciennes luttes syndicales, porte l'empreinte d'une double inspiration corporatiste et technocratique. L'exposé des motifs souligne la visée d'une efficacité accrue du travail au moyen d'une répartition rationnelle de la main-d'œuvre dont il déclare vouloir «améliorer la situation matérielle » et « relever le niveau moral ». Les dockers sont tenus, en contrepartie, de se présenter régulièrement à l'embauche. L'absence de solution à l'indemnisation des jours chômés continue néanmoins de faire reposer le coût de l'intermittence sur les seuls ouvriers. Laissée de côté en 1941, la question resurgit deux ans plus tard à Marseille où un «vœu » formulé par le père Loew propose en vain le versement d'une indemnité égale aux deux tiers du salaire de base ${ }^{15}$. Dans l'immédiat, la Fédération des Ports et Docks prend acte de la nouvelle loi. Par voie de presse, elle invite ses organisations à «faire désigner » leurs délégués dans les BCMO et préconise la mise sur pied d'un «régime spécial très souple» dans les ports dépourvus de bureau d'embauche ${ }^{16}$.

\section{La Charte, l'ultime créneau fédéral?}

\footnotetext{
${ }^{12}$ L'Atelier du 26 avril 1941.

${ }^{13}$ J. Berthelot, Sur les rails du pouvoir (de Munich à Vichy), Robert Laffont, 1968, p. 168.

${ }^{14}$ Cf. notre article : «Le statut des dockers de 1947 : acquis législatif et pratiques sociales », Cahiers de l'Institut régional du travail, Actes du colloque Construction d'une histoire du droit du travail, 20-21 septembre 2000, Aix-en Provence, 2001, pp. 241-259.

${ }^{15}$ M. R. Loew, Les dockers de Marseille. Analyse d'un complexe, L'Arbresle, Economie et humanisme, 1945, p. 79.

${ }^{16}$ Déclaration du 27 novembre du 13 décembre 1941. Au total, neuf BCMO seront créés avant la Libération.
} 
Créée par décret du 3 avril 1942, la «famille » des Transports est dotée, le 21 juillet, conformément à l'article 77 de la Charte du Travail, d'une commission provisoire d'organisation dont le bureau est nommé le 15 octobre. Au terme de longs échanges entre employeurs et salariés sur les contours de ses diverses sections, la famille comporte dix «branches ». La septième, dite des «activités auxiliaires des transports de marchandises », réunit cinq «professions », dont celle de la manutention, elle-même divisée, selon leur localisation, en trois «industries élémentaires » : ports maritimes, fleuves et chemin de fer. Avec Boucher, Dujardin et Le Gall, la Fédération des Ports et Docks domine la représentation des salariés des activités auxiliaires. Le 19 janvier 1944, de nouvelles nominations étoffent la commission des Transports transformée, le 24 août 1943, en comité social national provisoire.

Les choses traînent davantage pour les comités sociaux d'entreprise. Avec 99 CSE, les Transports se situent à un très médiocre $18^{\mathrm{e}}$ rang sur la trentaine de familles reconnues ${ }^{17}$. La faiblesse est plus marquée dans les ports en raison de la proportion élevée d'ouvriers intermittents. L'échelon intermédiaire des structures régionales prévues par la Charte peine davantage encore à prendre forme. Au printemps 1943, Le Gall évoque les difficultés rencontrées lors de la constitution, dans son fief normand, d'une sous-commission à Rouen. Ainsi doit-il «user de toute [son] autorité pour trouver des candidats » ${ }^{18}$. Au total, la représentation salariale des activités auxiliaires dans les sous-commissions régionales laisse à désirer alors que la CGT clandestine recommande, dès la fin 1943, « un sabotage complet et total de la Charte ${ }^{19}$. Hormis celle de Rouen et ses déclinaisons locales du Havre, de Caen et de Cherbourg, des militants des Ports et Docks siègent à Paris, Lille et Marseille. Absents de la sous-commission de Nice, ils refusent de siéger à Lyon et à Bordeaux après consultation des syndiqués. Au terme d'un vote serré, le comité national ne confirme pas moins, le 12 février 1944, le soutien fédéral à la Charte.

D'autres organismes voient le jour, à l'exemple de la sous-commission nationale d'entraide des transports à laquelle Le Gall demande de s'occuper des sinistrés, " seul moyen de démonter l'existence et l'utilité de la famille professionnelle ${ }^{20}$. Au double titre d'ancien prisonnier de guerre et de membre de la sous-commission havraise Hauguel reçoit la mission de procéder au reclassement professionnel des prisonniers rapatriés. Il va toutefois plus loin lorsqu'il accepte de participer, aux côtés de Vaillant et Quesnel, à la vie du Comité ouvrier de secours immédiats - COSI -, fondé en juin 1942 afin d'aider, dit-on, les sinistrés des bombardements avec le produit de la spoliation des Juifs. Á la Libération, les syndicalistes plaideront la réalité des secours délivrés, la mise à l'écart des groupuscules collaborationnistes et l'argument du moindre $\mathrm{mal}^{21}$. Plus réticents ou perspicaces, les militants rouennais mandatés par leurs camarades afin de «glaner des informations» sur le COSI, en démissionnent assez vite ${ }^{22}$.

\section{De nouvelles pratiques syndicales?}

Indépendamment des perspectives de survie sociale et des ambitions ouvertes à la faveur des événements, nombre de praticiens de la politique de présence sont convaincus d'agir au mieux des intérêts des travailleurs. A tous les niveaux, l'adhésion affichée à la Charte n'exclut pas, en effet, la revendication pour peu qu'elle n'emprunte pas la voie de l'action directe. Dans les colonnes de L'Atelier, Loriot énoncent les attentes des dockers:

\footnotetext{
${ }^{17}$ AN, F22 1973, statistique du 8 octobre 1943.

${ }^{18}$ AN, F22 1968, CPO, réunion du 16 avril 1943.

${ }^{19}$ La consigne sera reprise par une déclaration du bureau confédéral clandestin, en mai 1944.

${ }^{20}$ AN, F22 1973, séance du 3 juillet 1944.

${ }^{21} \mathrm{P}$. Aubery, «De l'isolement et de la misère à l'organisation professionnelle. Les dockers du port du Havre », La Révolution prolétarienne, $\mathrm{n}^{\circ}$ 352, juin 1951, p. 206.

${ }^{22}$ AICGTHS, Fonds Jayat, CNROS, lettres de Tate, Marchand et Carrier au Président de la Commission, les 17, 19 et 29 mai 1945 .
} 
locaux pour les ouvriers en attente d'une embauche, institution de délégués à la sécurité, promulgation d'un statut... Au besoin, le ton se fait véhément. «Les travailleurs des ports veulent vivre!", s'exclame-t-il, le 23 mai 1942, à propos du droit des intermittents à bénéficier de l'ensemble des lois sociales. Au Havre, la réduction d'un tiers du niveau des salaires entraîne une protestation du syndicat. Ses interventions répétées aboutissent au rétablissement des anciens tarifs le 4 avril 1941. Alors même que l'activité strictement portuaire se raréfie, des militants s'investissent dans des responsabilités étrangères aux quais, sinon aux dockers en cours de reconversion. Ainsi obligent-ils les employeurs à reverser la prime spéciale accordée par les Allemands aux ouvriers embauchés sur les chantiers du mur de l'Atlantique. La campagne déclenchée par Susini, au second semestre 1943, dans les colonnes de l'Émancipation nationale, l'hebdomadaire du PPF, procède d'autres préoccupations. D'un point de vue formel, le ton et les thèmes - conditions de travail, salaires, pouvoir d'achat, mise en cause du patronat et de l'administration, etc. - participent du syndicalisme. La démarche relève tout autant, cependant, de l'intimidation et du règlement de comptes en vue de conforter la mainmise sabianiste sur les quais marseillais.

Si la grève, interdite par la Charte, n'est plus à l'ordre du jour, la Fédération n'y renonce pas complètement. En 1942, elle diffuse ainsi la consigne de ne pas se présenter à l'embauche en réaction contre le refus d'étendre à tous les ports la prime accordée aux dockers de Paris, Marseille et Sète ${ }^{23}$. Suivi, le mot d'ordre aurait accéléré la promulgation d'un arrêté en ce sens.

Lieu de concertation, les commissions provisoires d'organisation offrent aussi une tribune à l'expression des «aspirations légitimes du monde ouvrier ${ }^{24}$. Au gré des causes, l'argumentaire oscille entre un corporatisme de bon aloi, critique envers l'intervention intempestive d'administrations incompétentes, et le registre plus rugueux du syndicalisme d'antan. Sans nier « la bonne volonté de certains patrons », Loriot constate que « d'autres [...] ne valent pas la corde pour les pendre ${ }^{25}$, tandis que Le Gall martèle : «je ne suis pas disposé pour ma part à faire ni la politique des trusts ni à éliminer complètement les petites entreprises ${ }^{26}$. La détermination est identique dans les sous-commissions régionales et locales. A Rouen, Carrier se heurte aux employeurs hostiles au regroupement des différentes catégories de salariés dans un syndicat dominé par les dockers. Mis en minorité, Carrier et ses amis claquent la porte. De retour, à la demande de la Fédération, les syndicalistes redisent leur détermination à ne rien voter sans consultation préalable de leurs mandants. Rompus aux rituels et procédures des négociations, les syndicalistes posent régulièrement le problème, non moins litigieux, des conditions de vie et de travail. Un mois après l'inauguration du comité social national des Transports, le secrétaire de la Fédération des Ports et Docks conteste les biais de la comparaison du pouvoir d'achat des salaires hebdomadaires de l'avant-guerre acquis en 40 heures à celui de revenus désormais calculés pour 48 heures de travail. Sur cette question cruciale, les syndicalistes font bloc face au patronat. Le Gall en fait un test de l'utilité du comité. Le conflit s'envenime devant les tergiversations gouvernementales et l'opposition des Allemands. Après une réunion séparée, les délégués ouvriers annoncent leur refus de siéger jusqu'à ce qu'une solution soit apportée à leurs revendications. Conscients du risque de rupture, mécontents des atermoiements ministériels, les employeurs et les cadres se rallient à l'ordre du jour des travailleurs... Le débat s'élargit pour déboucher sur une analyse critique des déséquilibres du dispositif chartiste. Ainsi Le Gall oppose-t-il les retards

\footnotetext{
${ }^{23}$ Rapport de Le Gall au congrès fédéral de 1946, op. cit., p. 9.

${ }^{24}$ AN, F22 1972, réunion du comité social régional de Rouen, le 18 avril 1944, intervention de Le Gall.

${ }^{25}$ L'Atelier du 12 avril 1941.

${ }^{26}$ AN, F22 1968, CPO, session du 24 février 1944.
} 
accumulés dans sa réalisation à la célérité de l'institution des comités d'organisation, instruments d'une puissance patronale sans précédent ${ }^{27}$.

Loin de Paris, le long de quais de plus en plus désertés par les navires de commerce et les ouvriers, chacun peut constater l'inexorable dégradation des conditions de travail et d'existence. L'allongement de la durée du travail décrétée en septembre 1939 réduit les perspectives d'embauche des intermittents. Le chômage partiel gagne le personnel mensualisé des entreprises dont certaines se résolvent à licencier. Selon Susini, le travailleur des ports, victime de «l'esprit de revanche » d'une partie du patronat, serait «plus maltraité qu'il ne l'a jamais été ${ }^{28}$. Á Marseille, il évalue à $50 \%$ la perte du pouvoir d'achat des contremaîtres dont beaucoup travaillent moins de 32 heures par semaine. Á Bordeaux, la participation, négociée avec le patronat local et votée par les syndiqués, du secrétaire du syndicat - Jarriod , au fonctionnement de l'Office de répartition de la main-d'œuvre vers les chantiers de la Kriegsmarine est diversement appréciée. Sommés de s'expliquer, à la Libération, sur cette «coopération ${ }^{29}$, les initiateurs et responsables de l'Office affirment s'être arrangés «pour raréfier la main-d'œuvre » et empêcher le départ de plusieurs dizaines de dockers au STO ${ }^{30}$. Ce dernier point ne va pas de soi. A Paris comme en Normandie, la critique se concentre sur les services rendus par les BCMO aux Allemands. Des ouvriers accusent le directeur du port de Rouen et les délégués du BCMO d'avoir prêté la main à la sélection des 100 dockers expédiés à Hambourg en novembre 1942. Pour leur défense, les responsables mis en cause limitent le rôle du bureau à l'affichage des listes et l'envoi des convocations... ${ }^{31}$ Ils contestent, en outre, l'interprétation donnée à leur présence lors de l'appel des requis, le jour de leur départ, et leur insistance à rappeler les «sanctions graves » encourues par les réfractaires.

\section{Conclusion : une expérience syndicale sans lendemain ?}

Dans une ultime défense et illustration de son attitude, Piquemal se résoudra à tenir tête à ses détracteurs de 1946. Plutôt que « de se mettre à l'ombre, de rester dans son coin, à critiquer, réplique-t-il, [...] moi, pendant quatre ans, je n'ai pas laissé courir. J'ai collaboré, si vous voulez, mais j'ai obtenu tout de même des avantages... $»^{32}$. Bien des militants des syndicats légaux se retrouvent dans ce plaidoyer. Beaucoup comprennent mal ou trop tard comment, de politique de présence en accommodement, ils ont franchi le seuil, irrémissible, de la compromission et de la complicité avec le régime de Vichy, voire avec l'occupant. Dans la continuité des rudes luttes de tendances et des compromis de l'entre-deux-guerres, forts des résultats acquis en matière d'organisation du travail et de reconnaissance de la profession de docker, ces syndicalistes ne démordent pas de leur conviction d'avoir servi du mieux qu'ils le pouvaient les intérêts ouvriers. Dans les conditions exceptionnelles de l'époque..., circonstances, précisément, que d'aucuns estiment atténuantes quand d'autres les jugent aggravantes.

L'opinion ouvrière, pour ce que l'on en sait, évolue dans le sens d'une prise de distance croissante. La reprise d'adhésions notée ici et là, à partir de septembre 1943, ignore les ports. L'effondrement des trafics n'explique pas tout. Embauché comme charbonnier sur les quais de Marseille, le père Loew note qu'après l'élimination de leurs «chefs authentiques », en 1939-1940, les dockers phocéens n'ont jamais accordé de crédit aux

\footnotetext{
${ }^{27}$ AN, F22 1972, séance du 3 décembre 1943.

${ }^{28}$ L'Émancipation nationale du 17 juillet 1943.

${ }^{29}$ AICGTHS, Fonds Jayat, CNROS, Rapport du comité d'épuration de l’UD de la Gironde, le 16 novembre 1944

${ }^{30}$ Idem, Note de M. Mallet au commissaire central, le 6 décembre 1944.

${ }^{31}$ Idem, p-v de la commission départementale, séances des 11 et 22 mai 1945.

${ }^{32}$ Intervention de Piquemal au congrès fédéral de 1946, op. cit., p. 53-58.
} 
hommes qui, «issus d'une bande politique bien connue [...], prétendaient les remplacer $»^{33}$. Dès avril 1941, Loriot signale le «risque » d'entendre les travailleurs parler de "réaction nationale », « comme les bons apôtres communistes ne manquent pas d'ailleurs de le colporter sous le manteau ${ }^{34}$. Contrer les «propagandes nocives et démagogiques des adversaires de toujours » ${ }^{35}$ apparaît plus ardu à la fin 1943. Les travailleurs, constate Le Gall, «viennent nous dire 'vous êtes dupes des autorités allemandes, des comités d'organisation et de l'entourage du Maréchal' ${ }^{36}$. «Les militants partisans de la Charte [...] se trouvent dans une situation impossible $»$, renchérit l'un de ses collègues ${ }^{37}$. La plupart persistent néanmoins à siéger activement jusqu'en juillet 1944 dans les organismes vichystes.

Il y a loin, de ce point de vue, entre le comportement du secrétaire de la Fédération et ce qu'en dit, en novembre 1945, un rapport de la Sécurité militaire qui voit en lui « un des organisateurs de la résistance $»^{38}$. Selon Saillant et Jayat, informés par Le Gall de ce qui se passait dans les organismes de la Charte, aurait averti de l'imminence d'une arrestation le militant qui, dans son bureau même, travaillait à la reproduction de circulaires clandestines ${ }^{39}$. Le secrétaire fédéral ne revendique pas, quant à lui, le titre de résistant, mais évalue à près de 800 le total des jeunes gens sauvés par ses soins d'un départ en Allemagne et mentionne ses mauvaises relations avec les Allemands contrariés de son refus d'adhérer au Comité d'informations ouvrières et syndicales. De là à prétendre qu'il " avait constamment refusé de propager la doctrine de la Charte» et «de donner les noms des camarades susceptibles de former les commissions ou sous-commissions $»^{40}$, il y a un pas que les archives interdisent de franchir. Malgré une évidente bienveillance, le président de la commission d'épuration syndicale conviendra du caractère « anormal » du long maintien de Le Gall dans les structures chartistes.

Que penser, alors, de l'itinéraire de Désiré Brest ? Militant unitaire de la batellerie exclu de la CGT en 1939, il quitte Rouen où il est trop connu pour naviguer entre le Nord et la région parisienne. Sans appartenir à la Résistance, il effectue des sabotages mineurs d'écluses ou de remorqueurs et se tient à l'écart des organisations de la Charte auxquelles il dissuade les mariniers d'adhérer. Pour un temps, car son nom apparaît, en 1944, au côté de celui d'autres militants communistes, parmi les administrateurs du syndicat unique de la navigation intérieure créé en janvier. Porté à sa direction, il rédige un projet de statuts débattu le 10 juillet, mais soutenu par Delval et Fischer ${ }^{41}$. Déjà membre, à la Libération, du comité de libération de l'ONN et de la commission de reconstitution des syndicats de la Seine, secrétaire général du syndicat de la marine marchande, le bureau confédéral le propulse au sommet de la Fédération des Ports et Docks où il rejoint Le Gall. Interpellé sur son action au syndicat unique, il assure s'être efforcé d'en saboter le fonctionnement, "ne sachant pas à l'époque combien de mois pouvait encore durer l'occupation ». Dans les deux cas, la commission d'épuration syndicale écarte toute sanction «pour insuffisance de preuves». Moins indulgents, les délégués au congrès fédéral de 1946 exigent d'autres comptes. Á l'issue d'un débat mouvementé soldé par le rejet du rapport de la direction sortante, Le Gall renonce à ses

\footnotetext{
${ }^{33}$ M. R. Loew, op.cit., p. 80.

${ }^{34}$ L'Atelier du 26 avril 1941.

${ }^{35}$ L'Atelier du 30 octobre 1943.

${ }^{36}$ AN, F22 1972, séance du 3 décembre 1943.

${ }^{37}$ Idem, intervention d'Huguet.

${ }^{38}$ Archives de la Préfecture de Police, dossier 63591. Note du capitaine Simonin, le 14 novembre 1945.

${ }^{39}$ AICGTHS, Fonds Jayat, CNROS, lettre de Saillant, le 25 juin 1945 et déclaration introductive de Jayat à la séance du 26 juin 1945.

${ }^{40}$ Idem, lettre de Le Gall à Jayat, le 27 novembre 1944.

${ }^{41}$ AN, F22 1846, CPO de la batellerie, séance du 10 juillet 1944.
} 
fonctions ${ }^{42}$. Sauvé in extremis, Brest reste au secrétariat fédéral en qualité d'adjoint permanent de Gagnaire, puis Baudin, avant d'occuper, entre 1962 et 1968, le poste de secrétaire général.

Par là, le congrès scelle la victoire des anciens unitaires sur les ultimes représentants de la direction en 1938. La condamnation prononcée contre le syndicalisme légal sous Vichy s'inscrit, par là, dans une temporalité qui déborde les années de guerre. Sa stricte application aux acteurs fédéraux les plus en vue affectera les relations compliquées qu'entretiendra Brest avec ses camarades de Parti. Ambiguë, elle laisse toutefois de côté de larges pans d'une expérience syndicale sans descendance reconnue, mais non sans legs.

\footnotetext{
${ }^{42}$ Placé par ses camarades du Havre à la tête du comité local intersyndical des organisations ouvrières du port, Le Gall recevra, en 1955, la médaille fédérale décernée aux anciens militants méritants.
} 